\section{Systemic Lupus Erythematosus - Basic, Applied, and Clinical Aspects}

George C. Tsokos, editor. Cambridge, Massachusetts, USA: Elsevier Academic Press, 2016, 640 pages, \$140 US

Systemic lupus erythematosus (SLE) is a complex autoimmune disease with many clinical manifestations that lead to significant morbidity and mortality. The textbook, Systemic Lupus Erythematosus - Basic, Applied, and Clinical Aspects, was written as a thorough examination of the historic, pathogenetic, clinical, and treatment dimensions of SLE. Each of these topics is divided into sections, with detailed chapters within each section. Each chapter was written by an international panel of experts in that particular topic as it relates to SLE.

The beginning of the text discusses the many theories surrounding the pathogenesis of SLE; specifically, the immunological derangements and how they affect the clinical symptoms seen in most patients. For those not well versed in the nuances of immunology, these chapters are thorough and exhaustive in detail, but comprehensible. A summary section is included at the end of each chapter and has a 2-fold purpose: as a great synopsis to highlight the important aspects of the chapter, or as a substitute for reading the entire chapter.
It is enjoyable to read the clinical pearls presented by the authors in relation to disease monitoring using the SLE Disease Activity Index and/or the British Isles Lupus Assessment Group index, as well as current and potential treatment options. The chapters dedicated to potential treatment options were well written - they connected the basic immunology discussed in earlier chapters to potential pharmaceutical targets.

This textbook is recommended because it is a valuable resource for beginning and seasoned rheumatologists. Additionally, it could serve as a basis for those engaging in basic science projects surrounding the pathogenesis of SLE.

DeANNA BAKER FROST, MD, PhD, Rheumatology Fellow, Medical University of South Carolina, Charleston, South Carolina, USA. Address correspondence to Dr. D. Baker Frost, Medical University of South Carolina, Department of Medicine, Division of Rheumatology and Immunology, 96 Jonathan Lucas St., Suite 908B-1, Charleston, South Carolina 29425, USA. E-mail: bakerde@musc.edu

J Rheumatol 2016;43:11; doi:10.3899/jrheum.160771 